\title{
Evaluación participativa para la gobernanza adaptativa de sistemas socio-ecológicos
}

\author{
Participatory Evaluation for Adaptative Governance of \\ Social-Ecologic System
}

\author{
Micaela Trimble ${ }^{1} y$ Ryan Plummer ${ }^{2}$
}

\begin{abstract}
Dentro del campo de la gobernanza adaptativa, la evaluación es un área que necesita mayor atención. La pesca artesanal en Uruguay fue el foco de esta investigación ya que está atravesando una transición de gobernanza. Consejos locales multiactorales han sido formados para buscar soluciones a problemas pesqueros en forma colectiva. En uno de estos consejos, facilitamos un proceso de evaluación participativa (EP) con involucramiento de diferentes actores implicados (pescadores e instituciones gubernamentales). El objetivo fue investigar el potencial de la EP para fortalecer Ios dos pilares principales del co-manejo adaptativo: la colaboración y el aprendizaje. Los resultados muestran que la cooperación y la comunicación entre los actores aumentaron; las relaciones pescadores-instituciones y las conexiones interinstitucionales mejoraron. Los talleres de evaluación se convirtieron en un espacio de aprendizaje; los actores mejoraron sus habilidades para participar e interactuar con otros. Como conclusión, la EP contribuye a la maduración del co-manejo adaptativo.
\end{abstract}

Palabras clave: Governanza; Co-manejo; Participación; Evaluación

Within the field of adaptive governance, evaluation has been identified as a knowledge void requiring attention. Small-scale fisheries in Uruguay were used as case study as they are going through a governance transition; local multi-stakeholder councils have been formed to look for solutions to fishery-related problems in a collective manner. In one of these councils, we facilitated a participatory evaluation process with involvement of different stakeholder groups (fishers and government agencies). The objective was to investigate the potential of participatory evaluation for enhancing the two main pillars of adaptive co-management: collaboration and learning. Findings show that cooperation and communication among stakeholders were enhanced. Both the relationships between fishers and government agencies, and the connections among agencies, were improved. The evaluation workshops became a learning space and stakeholders improved their skills to participate and interact with different actors.

\footnotetext{
${ }^{1}$ Universidad Brock, Canadá. Instituto Sudamericano para Estudios sobre Resiliencia y Sostenibilidad (SARAS), mica.trimble@gmail.com

2 Universidad Brock, Canadá
} 
2019| Tekoporá 1/ (1) Transformaciones territoriales en la región Este. Focus temático: transformaciones...

Participatory evaluation is thus an important process for the adaptative comanagement

Keywords: Governance; Co-management; Participation; Evaluation 


\section{INTRODUCCIÓN}

La gestión de recursos naturales actualmente reconoce que los recursos no están aislados en su ecosistema, sino que están entrelazados con componentes sociales, llevando al surgimiento del término "sistemas socio-ecológicos". Se trata de sistemas complejos, caracterizados por una interdependencia entre sus elementos humanos y sus elementos naturales, relaciones no lineales entre éstos, incertidumbres y escalas múltiples, con interacciones horizontales (entre elementos en un mismo nivel) y verticales entre ellas (Berkes et al., 2003). La complejidad para el manejo de estos sistemas se debe también a la diversidad de actores implicados (stakeholders), entre los que frecuentemente existen una pluralidad de valores y visiones sobre el sistema. La falta de previsibilidad de los sistemas socio-ecológicos resalta la importancia de eventos iterativos de "aprender haciendo" (learning by doing), típico del manejo adaptativo, de forma tal de adaptar los procesos de toma de decisiones de acuerdo a la variabilidad del sistema (Berkes et al., 2003). Este aprendizaje es clave para aumentar la capacidad de adaptación del sistema, para hacer frente tanto a la incertidumbre de la dinámica de los ecosistemas como a la interdependencia resultante de la complejidad del sistema social (Folke et al., 2005).

Esta nueva visión de la gestión de recursos naturales deja atrás el manejo gubernamental convencional, centralizado y jerárquico, también conocido como "comando y control" o "de arriba a abajo" (top-down management), para reconocer que una amplia participación de los usuarios de los recursos y otros actores implicados puede ayudar a solucionar la crisis de la gestión ambiental (Walker y Daniels, 2001; Berkes, 2010). La participación de diferentes actores, incluyendo organizaciones gubernamentales y no gubernamentales, comunidades locales y actores privados en los procesos de gestión, es un elemento central del concepto de gobernanza ambiental, que refiere a la forma en que son ejercidos el poder y las responsabilidades, cómo se toman las decisiones y qué voz tienen los ciudadanos y otros interesados (Lemos y Agrawal, 2006). La gobernanza ambiental puede ser también definida como el sistema de instituciones (incluyendo reglas, leyes, regulaciones, políticas y normas sociales) y organizaciones involucradas en gobernar el uso y/ o la protección de recursos naturales (Chaffin et al., 2014).

La gobernanza adaptativa es un tipo de gobernanza ambiental especialmente útil para lidiar con la incertidumbre y complejidad de los sistemas socio-ecológicos a través de la interacción dinámica entre actores, redes, organizaciones e instituciones (Chaffin et al., 2014). De creciente aplicación y análisis en la última década, la gobernanza adaptativa surge del interés social de manejar a los sistemas socio-ecológicos de forma holística tanto sea para aumentar la resistencia a cambios no deseados como para aumentar la habilidad del sistema de transformarse a un estado más deseado (Chaffin et al., 2014). El concepto de gobernanza adaptativa está muy relacionado con el de resiliencia, definida como la capacidad de un sistema socio-ecológico de absorber o incorporar disturbios naturales y humanos sin perder su estructura y su función, así como su habilidad de reorganizarse ante cambios abruptos (Folke, 2007). Diversos estudios de caso han mostrado que algunos aspectos importantes de la gobernanza adaptativa son la generación de conocimientos a partir de la combinación de conocimiento local y científico, la formación de redes y relaciones de confianza entre actores, la presencia de líderes que lleven adelante el proceso, las prácticas de manejo adaptativo a través de la reflexión y el aprendizaje, e instituciones flexibles en múltiples niveles (Folke et al., 2005). 
En este sentido, algunos autores plantean que la gobernanza adaptativa (Folke et al., 2005; Armitage et al., 2009) puede ser operacionalizada a través del co-manejo adaptativo, el cual combina la característica de aprendizaje dinámico típica del manejo adaptativo con la característica de conectar múltiples niveles, típica del co-manejo. Ambos términos (gobernanza adaptativa y co-manejo adaptativo) son frecuentemente usados como sinónimos (Chaffin et al., 2014). La deliberación analítica, involucrando a las diferentes partes en una discusión informada sobre reglas, fue identificada como un elemento clave para alcanzar una gobernanza adaptativa (Dietz et al., 2003). Asimismo, para que exista una gobernanza adaptativa, tiene que haber colaboración y redes sociales entre los actores, así como aprendizaje entre ellos, en los diferentes niveles y escalas del sistema (Folke et al., 2005; Pahl-Wostl et al., 2007; Chaffin et al. 2014).

Una de las tendencias en este campo de investigación, y que representa un área que resta ser explorada en profundidad, son los procesos y factores que ayudan a que se dé la transformación hacia una gobernanza o co-manejo adaptativos, o a sentar las bases para ello (Baird et al., 2014; Chaffin et al., 2014; Trimble y Berkes, 2015). La existencia de una legislación adecuada, de incentivos económicos y de organizaciones "puente" conectando diferentes niveles y escalas, son algunos de los elementos necesarios (Folke, 2007). Además, los componentes sociales del sistema tienen que estar adecuadamente preparados para que la transformación pueda tener lugar. Si bien es esencial que los diferentes actores implicados participen, la presencia de líderes y la construcción de confianza entre actores a nivel local determinan el surgimiento de la gobernanza adaptativa (Chaffin et al., 2014). Se ha planteado, además, que un cambio en el sistema hacia una gobernanza adaptativa requiere de "ventanas de oportunidad", como por ejemplo un cambio en las politicas, un aumento en las fuentes de financiación, o un desastre natural (Olsson et al., 2006). Otra de las tendencias en este campo de investigación trata sobre la evaluación de los procesos de gobernanza y co-manejo adaptativos, en parte para identificar conexiones causales entre los procesos y los resultados que éstos generan (Cundill y Fabricius, 2010; Plummer et al., 2012).

Legislación reciente en Uruguay ha marcado el inicio de ámbitos multi-actorales (de carácter consultivo o asesor) para la gestión de recursos naturales, representando una "ventana de oportunidad" en la transición hacia la gobernanza adaptativa. La nueva ley de pesca (№ 19.175) establece la conformación de estos ámbitos en dos niveles: (i) un Consejo Consultivo de Pesca, a nivel nacional, integrado por representantes de varios ministerios, empresarios, pescadores industriales, artesanales y otros trabajadores del sector, y (ii) Consejos Zonales Pesqueros para el co-manejo de los recursos en cada zona de pesca artesanal (definidas por la DINARA - Dirección Nacional de Recursos Acuáticos), integrados por representantes del gobierno nacional, departamental y municipal, y pescadores artesanales. Sin embargo, antes de la conformación de los consejos zonales se crearon Consejos Locales de Pesca para tratar problemas específicos en algunas localidades, representando entonces un tercer nivel, como en Ciudad de la Costa (Canelones) o Piriápolis (Maldonado). De los once consejos de pesca existentes a la fecha, seis están en la costa rioplatense y oceánica (San José, Montevideo, Ciudad de la Costa, Piriápolis, Punta del Este, La Coronilla-Barra del Chuy). Los Consejos Locales de Pesca fueron creados como ámbitos de información, intercambio de ideas y de trabajo entre los diversos actores vinculados al sistema pesquero artesanal. Son de carácter consultivo y tienen como objetivo promover el co-manejo pesquero, acercando a la DINARA con los representantes de pescadores y autoridades locales y/o nacionales. Son convocados por la DINARA y funcionan a partir de la realización de sesiones en las que se tratan temas propuestos por sus integrantes, vinculados a la pesca artesanal y al ordenamiento pesquero. También se discuten temas de carácter socioeconómico, ambiental y/o territorial. 
Según las características de la localidad, pueden participar otras instituciones u actores (por ejemplo, en Punta del Este participa la Dirección Nacional de Hidrografía y en Salto los pescadores deportivos).

La presente investigación tuvo como foco el Consejo Local de Pesca de la Costa (Canelones), primer consejo en la costa del Río de la Plata, en donde se facilitó un proceso de evaluación participativa con los cuatro grupos de actores involucrados (pescadores artesanales, DINARA, Prefectura y gobiernos municipales/departamentales) (Trimble y Plummer, 2018, 2019). Se trató de la primera instancia de evaluación. Además de buscar contribuir a mejorar el funcionamiento del consejo, el objetivo fue investigar el potencial del enfoque de evaluación participativa para propiciar los dos pilares principales del co-manejo adaptativo (colaboración entre actores y aprendizajes). Las etapas de una evaluación participativa, de forma resumida, incluyen las siguientes: (i) definir los fines u objetivos (por qué y para qué evaluar), (ii) identificar indicadores (qué evaluar), (iii) analizar el cumplimiento de los indicadores, (iv) reflexionar, intercambiar y discutir acciones a emprender. Estas diferentes etapas (parte de un ciclo adaptativo) son realizadas colectivamente por los diferentes actores. Este enfoque de evaluación, con el mismo origen que las investigaciones participativas, comenzó a utilizarse para evaluar proyectos de desarrollo y organizaciones comunitarias (Papineau y Kiely, 1996), para luego expandir su uso a proyectos de conservación e iniciativas de co-manejo o manejo colaborativo, incluyendo casos de humedales y áreas protegidas (Ferreyra y Beard, 2007; Sayer et al., 2007; Izurieta et al., 2011). Con las evaluaciones participativas se busca: mejorar la calidad del proceso evaluativo (ya que las personas involucradas realizan aportes en las diferentes etapas); aumentar la aplicabilidad de los resultados de la evaluación al estar siendo generados en el ámbito en que serán aplicados; fortalecer grupos u organizaciones compuestos por diferentes actores, y propiciar el aprendizaje colectivo entre los participantes (Papineau y Kiely, 1996; Estrella et al., 2000). Aunque este enfoque de evaluación ha sido ampliamente recomendado, las investigaciones empíricas sobre su alcance y resultados son escasas.

\section{ESTUDIO DE CASO Y MÉTODOS}

El desarrollo de estudios de caso es particularmente útil en investigaciones sobre evaluación de procesos (Yin, 1994). El estudio de caso para esta investigación fue el Consejo Local de Pesca de la Costa, el cual tuvo su sesión inaugural el 29 de octubre de 2012, a iniciativa de la DINARA en el marco del Proyecto GEF-DINARA-FAO "Ensayo piloto de un enfoque de ecosistemas para la pesca costera en Uruguay". Este Consejo de pesca se proponia analizar temáticas vinculadas a la pesca artesanal en Barra de Carrasco, Ciudad de la Costa y Neptunia. Hasta marzo de 2014 las sesiones del Consejo estuvieron a cargo de dicho proyecto. Cuando éste terminó, la Unidad de Pesca Artesanal de la DINARA pasó a estar a cargo del Consejo. Este "traspaso" conllevó algunas dificultades (por ejemplo, asociadas a los menores recursos materiales, financieros y humanos de la institución), haciendo que la convocatoria de las reuniones fuera interrumpida durante aproximadamente dos años. Entre octubre de 2012 y marzo de 2014 hubo ocho sesiones; la frecuencia de reuniones fue mensual, bimensual 0 semestral. Luego que el proyecto terminara y hasta fines de 2016, hubo tres sesiones (julio de 2014, setiembre y noviembre de 2016). Los talleres de la evaluación participativa del Consejo (que se describen a continuación) tuvieron lugar en el período en el cual no hubo sesiones.

En las reuniones de la etapa inicial del Consejo de Pesca (2012-2014), que tenían lugar en el Municipio de Ciudad de la Costa, se encontraban los representantes de los pescadores para Ciudad de la Costa y Neptunia, con representantes de DINARA, Prefectura, Intendencia de 
Canelones y Municipios de Paso Carrasco, Ciudad de la Costa y Salinas. Otros pescadores de estos sitios y de otras localidades participaron de algunas sesiones, así como referentes de otras instituciones o grupos. Entre diciembre de 2014 y noviembre de 2015, se facilitaron cuatro talleres de trabajo (de aproximadamente tres horas de duración) en los que los integrantes del consejo identificaron los fines de la evaluación, definieron los indicadores o aspectos relevantes para analizar el consejo y reflexionaron de forma conjunta sobre el funcionamiento de éste. El número de participantes en los talleres varió entre cuatro y once; no todos los grupos de actores (DINARA, Prefectura, gobiernos locales, pescadores) estuvieron presentes en todos los talleres.

Los integrantes del Consejo de Pesca identificaron varios aspectos positivos de este ámbito de encuentro de diferentes actores, como por ejemplo, la formación de vínculos nuevos y el buscar soluciones a problemas de la pesca. Pero también reconocieron varias debilidades 0 desafíos que refieren al funcionamiento del consejo, al poco avance en ciertos temas y a la baja capacidad para concretar acciones, en parte debidos a carecer de acuerdos claros de trabajo. Relacionado con esto, los integrantes del Consejo plantearon como expectativas para la evaluación participativa: ser autocríticos; mejorar el consejo tras poner sobre la mesa aciertos y errores; dar seguimiento a los temas y recabar distintas miradas.

Para iniciar el proceso de la evaluación, los participantes identificaron indicadores (variables, factores, dimensiones) que refieren a aspectos cualitativos y cuantitativos con los que se puede analizar el Consejo (Trimble y Plummer, 2018). Éstos pueden dividirse en dos grandes categorías: aquellos que buscan evaluar aspectos relacionados con la participación de los diferentes actores y aquellos que se proponen para evaluar los resultados del Consejo (Tabla 1). Varios de los indicadores referidos a las reuniones están vinculados a las dificultades encontradas y refuerzan la propuesta de los participantes de definir acuerdos de funcionamiento (por ejemplo, sobre la convocatoria y la frecuencia de las reuniones). Por otra parte, los indicadores propuestos para evaluar los resultados del consejo están estrechamente vinculados con las expectativas que los distintos actores manifestaron sobre éste, como la mejora de los recursos pesqueros, la mejora de la calidad de vida de los pescadores y la mejora de las relaciones entre pescadores e instituciones. Sin embargo, los participantes también reconocieron que algunos de los resultados esperados, como la mejora del estado de los recursos pesqueros, escapan a la escala del Consejo de Pesca, que es local.

Tabla 1. Indicadores o aspectos propuestos por los integrantes del Consejo Local de Pesca de la Costa para su evaluación

\begin{tabular}{|c|c|}
\hline $\begin{array}{l}\text { (1) Indicadores para evaluar la participación } \\
\text { en el consejo }\end{array}$ & $\begin{array}{l}\text { (2) Indicadores para evaluar los resultados del } \\
\text { consejo }\end{array}$ \\
\hline $\begin{array}{l}\text { 1.1. Convocatoria (con anticipación) y } \\
\text { concreción de las reuniones }\end{array}$ & $\begin{array}{l}\text { 2.1. Mejora en la administración de los } \\
\text { recursos pesqueros }\end{array}$ \\
\hline 1.2. Frecuencia de reuniones & $\begin{array}{l}\text { 2.2. Mejora en el estado de los recursos } \\
\text { pesqueros }\end{array}$ \\
\hline $\begin{array}{l}\text { 1.3. Representatividad (participación de } \\
\text { representantes idóneos o adecuados de las } \\
\text { instituciones y pescadores) }\end{array}$ & 2.3. Mayor cuidado de la faja costera \\
\hline 1.4. Continuidad de actores & $\begin{array}{l}\text { 2.4. Mejora en la calidad de vida de los } \\
\text { pescadores }\end{array}$ \\
\hline
\end{tabular}




\subsection{Participación de otras instituciones según 2.5. Mayor organización y formalización de los el tema a tratar pescadores}

1.6. Reuniones centradas en los temas que el consejo elige

2.6. Mayor cumplimiento de las funciones de cada institución del consejo

1.7. Participación de todos los integrantes del consejo durante el tratamiento de los temas

1.8. Metas y resoluciones en cada reunión del consejo, registradas en actas
2.7. Mejora de las relaciones entre pescadores e instituciones

2.8. Soluciones a problemas de la pesca artesanal

2.9. Mayor seguridad en la navegación y en el cumplimiento de los procedimientos para hacerse a la mar

2.10. Propuestas del consejo que son tomadas en cuenta por la DINARA y las demás instituciones

2.11. Consultas que DINARA hace al consejo antes de emitir nuevas reglamentaciones

A fin de investigar el proceso y alcance de la evaluación participativa considerando el marco conceptual del co-manejo adaptativo (Trimble y Plummer, 2019), se emplearon los siguientes métodos para la recolección de información (Yin, 1994; Berg, 2004): entrevistas semiestructuradas con participantes; observación participante durante las sesiones del consejo y durante los talleres de evaluación y análisis de documentos (actas y otros documentos generados en dichos ámbitos). Específicamente, para investigar el proceso y los resultados de la evaluación participativa, se desarrollaron dos rondas de entrevistas individuales con los actores involucrados, una en medio del proceso de evaluación (julio-agosto de 2015, con once participantes) y otra tras haber finalizado éste (febrero-mayo de 2017, con cinco participantes los restantes no tenían disponibilidad para ser entrevistados o para completar un formulario electrónico). Se realizaron preguntas abiertas y cerradas para indagar en los cambios ocurridos en las relaciones entre los participantes (comunicación, cooperación, confianza), como consecuencia del proceso de evaluación participativa y en los aprendizajes percibidos (conocimientos nuevos, habilidades, cambios de actitud). Las transcripciones fueron codificadas de forma inductiva y deductiva, utilizando el software Atlas.ti. La triangulación de métodos y fuentes de información contribuyó a la validez de los resultados (Conley y Moote, 2003).

\section{RESULTADOS}

\section{EVALUACIÓN PARTICIPATIVA PARA LA PROMOCIÓN DE LA COLABORACIÓN ENTRE ACTORES}

Participantes de los cuatro grupos de actores señalaron que la cooperación y la comunicación entre ellos mejoraron durante la evaluación participativa del Consejo de pesca. Los resultados de las entrevistas también mostraron que las relaciones que ya existían entre los actores participantes se volvieron más próximas (por ejemplo, entre DINARA y los gobiernos locales, entre DINARA y Prefectura, entre Prefectura y gobiernos locales, entre pescadores y gobiernos locales). Ya en la mitad de la evaluación participativa, la mayoría de los 
entrevistados señaló que los talleres les habían permitido conocer mejor a otros participantes; los ejemplos más comunes para ilustrar eso fueron las relaciones entre pescadores y las diferentes instituciones. Por ejemplo, el representante de la DINARA consideró que: "El relacionamiento más importante dentro de los Consejos para DINARA, es con los pescadores y en esa instancia conocí a muchos y creo que una de las funciones del Consejo y de estos talleres es ir conociéndonos cada vez más y saber más o menos planteos que cada uno tiene para hacer".

Sin embargo, no se formó un grupo multiactoral sólido en el proceso de la evaluación participativa del Consejo; los actores no iniciaron ninguna acción conjunta como grupo. Además, si bien las relaciones se tornaron más próximas, la confianza solo aumentó entre algunos actores participantes, como por ejemplo entre la DINARA y los gobiernos locales (Figura 1) y los conflictos existentes (por ejemplo, entre pescadores y DINARA o Prefectura) no fueron resueltos. Según explicaron los entrevistados, la confianza aumentó debido a la interacción cara a cara en los talleres y a través de conocer la realidad y el trabajo de los demás actores.

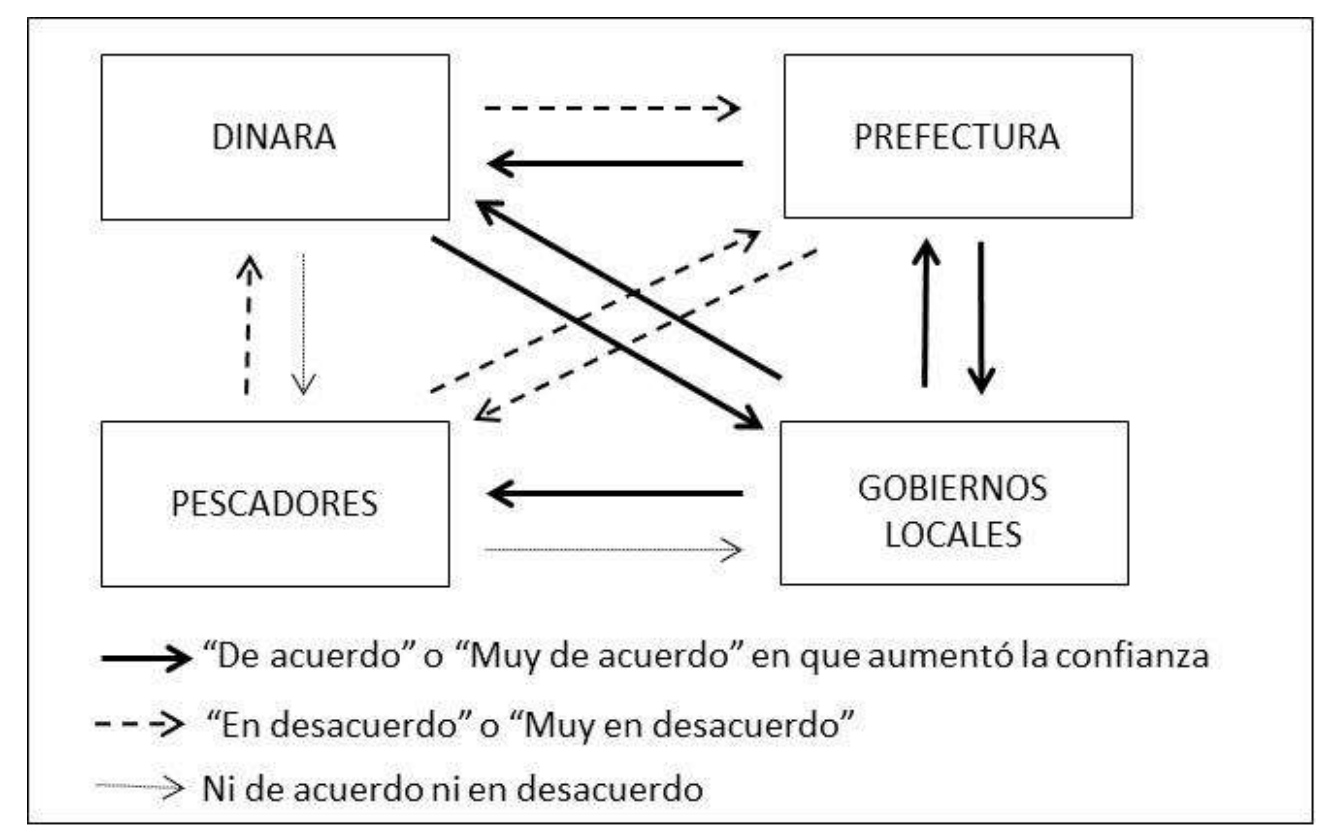

Figura 1. Cambios en la confianza entre los actores participantes, como consecuencia de la evaluación participativa del Consejo de Pesca. (Elaboración propia a partir de las respuestas de los entrevistados a una pregunta de múltiple opción realizada tras finalizar la evaluación participativa. (Modificado de Trimble y Plummer, 2019).

\section{EVALUACIÓN PARTICIPATIVA COMO PROCESO GENERADOR DE APRENDIZAJE}

Todos los participantes identificaron a la evaluación participativa como un proceso de aprendizaje. De hecho, sus expectativas principales fueron mejorar el funcionamiento del Consejo de pesca a través de corregir errores y aprender de éxitos y fracasos. Los aprendizajes identificados variaron según los actores (ver a continuación) pero todos coincidieron en que la evaluación participativa los ayudó a entender la perspectiva de los demás y a mejorar sus habilidades para interactuar con otros actores. Hubo respuestas contradictorias sobre las 
contribuciones de la evaluación participativa en la construcción de una visión común del Consejo, y en el aumento de su flexibilidad y capacidad para responder ante desafíos.

Con relación a los aprendizajes, los pescadores explicaron que durante la evaluación participativa aprendieron a comunicarse con otros actores y a involucrarse activamente en conversaciones sobre tópicos diversos. A modo de ejemplo, uno señaló: “Aprendés cosas nuevas porque estás en contacto con gente que está en otros temas, no de la pesca, que es de lo único que sabemos hablar nosotros, de pescado y de agua." Integrantes de la Prefectura y de los gobiernos locales tuvieron numerosos aprendizajes sobre la pesquería, incluyendo aspectos sociales, ecológicos e institucionales.

Como producto del aprendizaje colectivo en los talleres, se destaca una serie de acuerdos de funcionamiento propuestos por los participantes para superar las debilidades que el consejo estaba enfrentando:

Convocatoria: cualquiera de los integrantes del consejo puede convocar una reunión cuando lo amerita (por ejemplo, cuando hay un problema). Las reuniones se deben convocar con al menos una semana de anticipación.

Frecuencia de reuniones: tener 3 reuniones ordinarias por año y reuniones extraordinarias cuando se necesiten.

Representatividad: cada representante en el consejo debe ser representativo de su grupo, colectivo o institución. Debe haber sido elegido o designado para tal tarea; concurrir preparado a las reuniones; consultar a su grupo o institución para tomar decisiones; trasmitir a su colectivo lo resuelto en el consejo. Se propuso incorporar una revisión de los representantes para mantenerlos o cambiarlos (por ejemplo, cada 2 años).

Continuidad de actores: siempre que sea posible debe participar la misma persona en el consejo. Cuando no puede concurrir, debe ir su suplente, quien debe estar siempre informado de lo que se ha tratado en reuniones anteriores. Si un representante se da cuenta que no puede cumplir con el compromiso asumido, se debe elegir a otra persona.

Comunicación: los integrantes del consejo deben estar continuamente comunicados por diferentes medios (e-mail, mensaje de texto, a través de Prefectura, pizarrón de la cooperativa de pescadores, web de DINARA, etc.).

Actores participantes: el Consejo de Pesca de la Costa debería incluir a representantes de los pescadores del Arroyo Pando y de San Luis. Si bien los representantes son los que tendrán voto en caso de tomar una decisión, en las reuniones podrán participar otros pescadores. Se invitará a otras instituciones a participar de las reuniones cuando estén implicadas en los temas a tratar.

Desarrollo de las reuniones: los temas a tratar serán propuestos por los integrantes del consejo y se establecerá un orden del día antes de la reunión. Al finalizar la reunión se definirán los temas para la próxima. Todos los participantes deben saber de antemano los temas a tratar. Se puede proponer otros temas al iniciar la reunión y de no tratarse ese día, quedarán para la siguiente. Se sugirió establecer un tiempo determinado para tratar cada tema y para iniciar y finalizar la reunión.

Participación y moderación de las reuniones: todos los integrantes del consejo deben participar en la discusión de los temas. Se puede establecer un tiempo límite por intervención. El moderador debe invitar a los que hablan poco a que den su opinión y debe tratar que otros no hablen de más. Si hubiera participación de otras personas además de los representantes, se regularán sus intervenciones para que el tratamiento de los temas no se extienda demasiado. El rol de moderador de las reuniones puede ser rotativo. En caso de tener temas complicados 0 
controversiales a ser tratados, se sugirió considerar la participación de un moderador externo (o sea, que no sea integrante del consejo).

Actas: son elaboradas por un secretario de actas y se distribuyen de forma electrónica y en papel (los Municipios colaborarán en la impresión y distribución). Las actas deben mantener informados a los integrantes del consejo (titulares y suplentes) de los temas que se tratan. También constituyen una fuente de información a la hora de analizar las reuniones anteriores (temas tratados, participación de representantes, etc.).

División de tareas: se formará una secretaría del consejo entre DINARA y pescadores para hacer las citaciones, definir fechas, hacer actas, manejar información de permisos, entre otras actividades.

Impacto o alcance de las decisiones del consejo: todas las propuestas que el consejo envíe a las instituciones deberían ser analizadas por éstas. Se espera que al menos un mínimo de las propuestas sea aceptado. Cuando las instituciones no tomen en cuenta las sugerencias 0 propuestas del consejo, deberían explicar los motivos. Aunque el consejo no sea vinculante (lo que el consejo proponga no es necesariamente lo que DINARA o el MGAP van a decidir), DINARA debería generar instancias de información, intercambio y consulta antes de emitir nuevas reglamentaciones para la zona.

Difusión del consejo: se debe informar a otras instituciones sobre lo actuado por el consejo para que tengan conocimiento de éste. Para ello se propuso elaborar una base de datos con información de las competencias y los referentes de las diferentes instituciones. Las acciones del consejo también deben ser comunicadas a los vecinos, usando por ejemplo medios de prensa locales. Además, las actas del consejo deberían estar disponibles para todos los interesados (por ejemplo, a través de la web de DINARA y Municipios).

Seguimiento y evaluación: se sugirió conformar un equipo o comisión de seguimiento para asegurar que se dé continuidad a los temas y para evaluar las reuniones del consejo. Se propuso incorporar una evaluación continua del consejo y una evaluación cada cierto tiempo (por ejemplo, una vez al año).

\section{CONCLUSIONES}

A modo de conclusión, esta investigación demuestra que es posible incorporar de forma sistemática un mecanismo de evaluación en la transición hacia una nueva forma de gobernanza (en este caso, de la pesca artesanal en Uruguay). La evaluación participativa contribuyó a fortalecer los dos pilares principales del co-manejo adaptativo, la colaboración entre los actores y el aprendizaje colectivo, aunque con algunas limitaciones (Trimble y Plummer, 2019). Los resultados del presente trabajo son relevantes tanto para los consejos de pesca como para otros ámbitos de gobernanza referidos a recursos naturales y planificación y ordenamiento territorial. Las evaluaciones participativas permiten mejorar la calidad del proceso evaluativo con los aportes de los implicados, y aumentar la aplicabilidad de los resultados de la evaluación, entre otros. Dado que el co-manejo y la gobernanza son procesos a largo plazo, incorporar espacios de monitoreo y evaluación es importante para propiciar la reflexión y la identificación de estrategias para mejorar las prácticas que se desarrollan.

\section{AGRADECIMIENTOS}

La primera fase de esta investigación fue financiada por el Centro Internacional de Investigaciones para el desarrollo a través de su programa "Canada-Latin America and the Caribbean Research Exchanges (IDRC-LACREG)". La segunda fase fue financiada por el Centro de Investigaciones en Sustentabilidad Ambiental (ESRC) de la Universidad de Brock (Canadá), a través de una beca de postdoctorado a M. Trimble. 
También se recibió apoyo de CAPES - Ministerio de Educación Superior de Brasil. Marila Lázaro, Patricia Iribarne y Karina Dracott colaboraron con la organización y desarrollo de los talleres de la evaluación participativa. La investigación contó con la aprobación de los Comités de Ética de las Universidades de Brock y Manitoba. El interés y compromiso de los participantes en esta investigación (integrantes del Consejo Local de Pesca de la Costa) también merecen ser reconocidos.

\section{BIBLIOGRAFÍA}

Armitage, D. R., et al. (2009). Adaptive co-management for social-ecological complexity. En: Frontiers in Ecology and the Environment, 7, 95-102.

Baird, J., Plummer, R. y Pickering, K. (2014). Priming the governance system for climate change adaptation: the application of a social-ecological inventory to engage actors in Niagara, Canada. En: Ecology and Society, 19(1), 3. http:// dx.doi.org/ 10.5751/ ES-06152-190103

Berg, B.L. (2004). Qualitative Research Methods for the Social Sciences. Boston: Pearson.

Berkes, F., Colding, J. y Folke, C. (eds). (2003). Navigating social-ecological systems: Building resilience for complexity and change. Cambridge: Cambridge University Press.

Berkes, F. (2010). Devolution of environment and resources governance: trends and future. En: Environmental Conservation, 37(4), 489-500.

Chaffin, B. C., Gosnell, H. y Cosens, B. A. (2014). A decade of adaptive governance scholarship: synthesis and future directions. Ecology and Society, 19(3), 56. http://dx.doi.org/ 10.5751/ES$\underline{06824-190356}$

Conley, A. y Moote, M. A. (2003). Evaluating collaborative natural resource management. Society and Natural Resources, 16, 371-386.

Cundill, G. y Fabricius, C. (2010). Monitoring the governance dimension of natural resource comanagement. En: Ecology and Society, 15. http:// www.ecologyandsociety.org/ vol15/ iss1/ art15/

Dietz, T., Ostrom, E. y Stern, P. C. (2003). The struggle to govern the commons. En: Science, 302, 1907-1912.

Estrella, M., et al. (eds). (2000). Learning from change. Issues and experiences in participatory monitoring and evaluation. IDRC, Ottawa, and Intermediate Technology Publications, London.

Ferreyra, C. y Beard, P. (2007). Participatory Evaluation of Collaborative and Integrated Water Management: Insights from the Field. En: Journal of Environmental Planning and Management, 50(2), 271-296.

Folke, C., et al. (2005). Adaptive governance of social-ecological systems. En: Annual Review of Environment and Resources, 30, 441-73.

Folke, C. (2007). Social-ecological systems and adaptive governance of the commons. En: Ecological Research, 22, 14-15. 
Izurieta, A., et al. (2011). Developing indicators for monitoring and evaluating joint management effectiveness in protected areas in the Northern Territory, Australia. En: Ecology and Society, 16(3), 9. http:// dx.doi.org/ 10.5751/ ES-04274-160309

Lemos, M. C. y Agrawal, A. (2006). Environmental governance. En: Annual Review of Environment and Resources, 31, 297-325.

Olsson, P., et al. (2006). Shooting the rapids: navigating transitions to adaptive governance of social-ecological systems. En: Ecology and Society, 11(1), 18. http:// www.ecologyandsociety.org/ vol11/ iss1/art18/

Pahl-Wostl, C., et al. (2007). Managing change toward adaptive water management through social learning. En: Ecology and Society, 12(2), 30. http:// www.ecologyandsociety.org/ vol 12/ iss2/art30/

Papineau, D. y Kiely, M. (1996). Participatory evaluation in a community organization: fostering stakeholder empowerment and utilization. En: Evaluation and Program Planning, 19(1), 19-93.

Plummer, R., et al. (2012). Adaptive comanagement: a systematic review and analysis. En: Ecology and Society, 17(3), 11. https:/ / www.ecologyandsociety.org/ vol17/ iss3/ art11/

Sayer, J., et al. (2007). Assessing environment and development outcomes in conservation landscapes. En: Biodiversity and Conservation, 16(9), 2677-2694.

Trimble, M. y Berkes, F. (2015). Towards adaptive co-management of small-scale fisheries in Uruguay and Brazil: lessons from using Ostrom's design principles. En: Maritime Studies, 14, 14. http:// dx.doi.org/ 10.1186/ s40152-015-0032-y

Trimble, M. y Plummer, R. (2018). Participatory evaluation in times of governance transition: the case of small-scale fisheries in Uruguay. Ocean \& Coastal Management, 161, 74-83.

Trimble, M. y Plummer, R. (2019). Participatory evaluation for adaptive co-management of social-ecological systems: a transdisciplinary research approach. Sustainability Science, 14(4), 1091-1103.

Walker, G. B. y Daniels, S.E. (2001). Natural resource policy and the paradox of public involvement: Bringing scientists and citizens together. En: Understanding Community-based Ecosystem Management (Gray, G. J., Enzer, M. J. and Kusel, J., eds). The Haworth Press, Inc., New York., 253-269.

Yin, R. K. (1994). Case Study Research. Design and Methods. 2nd Ed. Applied Social Science Research Methods Series Vol 5. Sage Publications Inc., Thousand Oaks, California. 